\title{
Experimental Study of Plused arc Machining for Superalloy GH4169
}

\author{
Dongmei Zhao ${ }^{1, a^{*}}$ and Chenguang Zhu ${ }^{1, b}$ \\ ${ }^{1}$ Engineering Training Center Xinjiang University Urumqi, China \\ aZDM1925@163.com, ${ }^{\mathrm{b} D a n i e l z h u 86 @ 126 . c o m ~}$ \\ *The corresponding author
}

\begin{abstract}
Keywords: Pulsed arc machining; Nickel base superalloy; Recast layer
\end{abstract}
\begin{abstract}
Pulsed arc machining can be used to process the difficult-to-machining materials such as nickel base superalloy with high efficiency, but the surface processing quality is not unsatisfactory. To improve the processing quality, surface micro topography, recast layer were observed in the pulsed arc machining of GH4169 superalloy, surface roughness and microhardness has been tested herein. The results shows that processing parameters in duty cycle at $15 \sim 55 \%$, pulse frequency at $400 \sim 700 \mathrm{HZ}$ achieved surface roughness lower than $25 \mu \mathrm{m}$. The recast layer that generated in process result in work harden within $0 \sim 50 \mu \mathrm{m}$ from the surface. Cracks were easily formed by high frequency and duty cycle, the increased thickness of recast layer formed by low frequency. The machining quality can be improved by reasonable parameters selected.
\end{abstract}

\section{Introduction}

Superalloy are widely used in the aerospace and nuclear industries[1],GH4169 is a Nb precipitation strengthened nickel-based superalloy, the microstructure of GH4169 consists of the $\gamma$ matrix, the face-centred cubic $\gamma^{\prime}$ phase and the body-centred cubic $\gamma^{\prime \prime}$ phase[2]. It has not only excellent mechanical properties, such as high yield strength, corrosion , oxidation resistance and high-ductility, which exhibits good performance in the temperature range from $-253^{\circ} \mathrm{C}$ to $700^{\circ} \mathrm{C}$, but also good formability and weldability[3].Because of its low thermal conductivity, during the manufacturing process high cutting temperature, serious tool wear and work hardening, and the processing efficiency is very low, make it become the typical difficult to machine materials[4].

Pulse arc machining, or short-arc machining, is a non-traditional manufacturing process that removes conductive material from the workpiece by the eroding effect of a series of recurring current pulsed arc discharges between two electrodes. The characteristics of pulse arc machining are it does not creat stresses in the work material since the tool never comes in contact with the work; theoretically, any material that is electrically conductive can be processed with high machining efficiency(material removal rate reach to $1.92 \times 10^{5} \mathrm{~mm}^{3} / \mathrm{min}$ ), small work noise, regardless of its hardness and strength,. It is extremely suitable for superhard materials such as cemented carbide, which are extremely difficult to cut by traditional methods [5].

There is extensive research about machining of nickel-based superalloy by scholars. W.Zhao use BEAM machining Inconel718 achieved MRR exceeds $11,300 \mathrm{~mm}^{3} / \mathrm{min}$, and the tool wear ratio is lower than $1 \%[6]$. Fuzhu Han investigated High-speed EDM milling with moving electric arcs achieved high material removal rate and good machining accuracy[7].Dinesh Thakur optimized high speed turning of Inconel718 parameters with Taguchi method, the experiment obtained the lower cutting force by optimized cutting speed and feed combination[8].S Dhanabalan optimization of machining parameters of EDM machining Inconel718 by Taguchi method obtained good results[9].

In this paper, a preliminary study using pulsed arc machining superalloys, discusses the effects of electric parameters on the machining surface quality, provide a theoretical basis for improving the quality of pulsed arc machining. 


\section{Experimental Procedures}

Test Materials and Processing Conditions. Nickel-based superalloy GH4169 is selected as the sample material, its chemical composition is shown in Table 1[10]. Sample is cut into sizes of $15 \mathrm{~mm} *$ $25 \mathrm{~mm} * 45 \mathrm{~mm}$ by DK7740 EDM.

Table 1 Elemental composition of GH4169 (wt.\%)

\begin{tabular}{|c|c|c|c|c|c|c|c|c|}
\hline $\mathrm{C}$ & $\mathrm{Cr}$ & $\mathrm{Ni}$ & $\mathrm{Mo}$ & $\mathrm{Nb}$ & $\mathrm{Co}$ & $\mathrm{Ti}$ & $\mathrm{Al}$ & $\mathrm{Fe}$ \\
\hline$<0.08$ & $17.0 \sim 21$. & $50.0-55.0$ & $2.80 \sim 3.3$ & $4.75 \sim 5.5$ & $\leq 1.0$ & $0.75-1.1$ & $0.30 \sim 0.7$ & $19 \sim 20.0$ \\
& 0 & & 0 & 0 & & 5 & 0 & \\
\hline
\end{tabular}

DHZK6330 experimental pulsed arc CNC machine tools as the test device. The system including processing power, cathode means, anode means, and mixed media injection devices. Energy provided by CTKP-36/2000FN type FM pulse power, the output voltage range $3 \sim 36 \mathrm{~V}$, the output peak current can reach to 2000A. Working medium is gas-liquid mixture. When processing the workpiece connected positive power, the tool connected negative power, tool and workpiece to maintain a certain discharge gap and continued injecting working medium, the working medium take away the erosion from the processing area, Continuously impulsive arc discharge is created between the electrodes, intese heat is created in the localized area of the arc impact, the molten metal is expelled from the surface of the workpiece. The processing parameters of pulsed arc machining is shown in Table 2.

Table 2 Machining parameters

\begin{tabular}{|c|c|}
\hline Reference voltage(V) & $16 \mathrm{~V}$ \\
\hline Pulse frequency(HZ) & $100 \sim 900$ \\
\hline Electrode & Workpiece(+), tool(-) \\
\hline Duty ratio(\%) & $15 \sim 95$ \\
\hline Feed rate(mm/s) & $0.02 \mathrm{~mm} / \mathrm{r}$ \\
\hline Pneumatic, hydraulic & $0.1 \mathrm{MPa}$ \\
\hline
\end{tabular}

Measurement Method of Surface Integrity. Surface roughness measured by TR210 roughness measure instrument, Choose five sampling points in measurement, the sampling length is $0.25 \mathrm{~mm}$, the range is $80 \mu \mathrm{m}$.

To observe the change of the microstructure in HAZ of grain, Making the specimen vertical to the finished surface, prepare metallography sample, grinding cutting section, corrosion after polishing, the etchant formulations is: $\mathrm{H}_{2} \mathrm{SO}_{4}(5 \mathrm{ml}), \mathrm{HCL}(150 \mathrm{ml}), \mathrm{CuSO}_{4}(20 \mathrm{~g}), \mathrm{H}_{2} \mathrm{O}(80 \mathrm{ml})$; Micro-hardness measurements using the HXD-1000TB Video Digital Vickers hardness, punching from the surface to the inside every $50 \mu \mathrm{m}$ in sequencer, and observe the microstructure.

\section{Results and Discussion}

Surface Morphology. Choose the machining parameters of sample at voltage 16V and frequency $400 \mathrm{HZ}$ cutting by WEDM machining, using ultrasonic $15 \mathrm{~min}$ to remove surface stains, observed by scanning electron microscopy, the results shown in Fig. 1.

As shown in Fig.1a, the surface of pulse arc machining having distinct peaks and valleys, while distribution of plurality of irregular spherical droplet at random, the diameter of droplet is about 5 $45 \mu \mathrm{m}$, which is affected by the force of the explosion in the pulsed arc discharge, fly out from the discharge region after sticking on the machined surface is formed after condensation. At the same time there are many micropores exist in processing surfaces, which is the discharge process of pulsed arc discharge can produce large amounts of gas, and the gas is dissolved in the bath reaches saturation, when the molten metal cools and re -solidification in the machined surface, the transition from liquid to solid process makes the gas solubility mutation, created a condition as the gas evolution, resulting surface has many pores (Fig.1b). 
There are surface microcracks exists in some area (Fig.1c), compare the material surface morphology on different duty cycles, with the duty ratio increase, a substantial increase in the number of surface crack (Fig.1d). This is because the process of pulse arc machining, melting rapid at high temperature, even gasification, the molten metal rapid cooling by the liquid working.

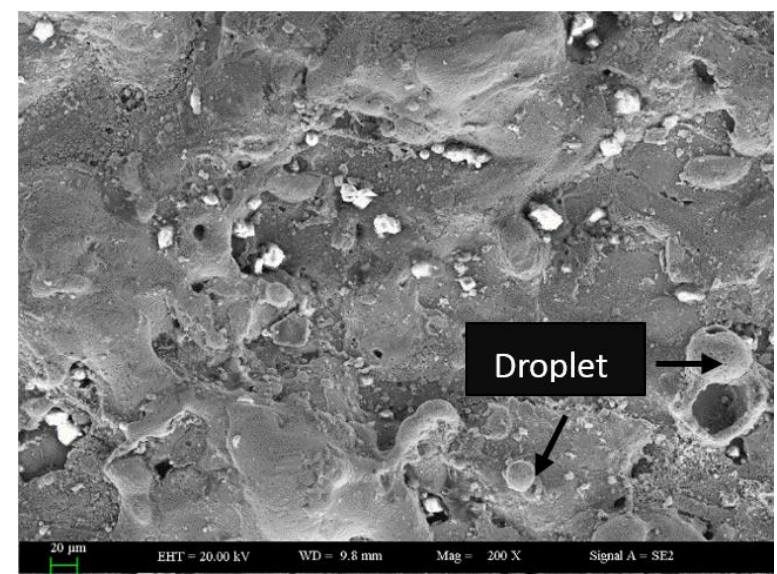

a) $\mathrm{D}=50 \%$

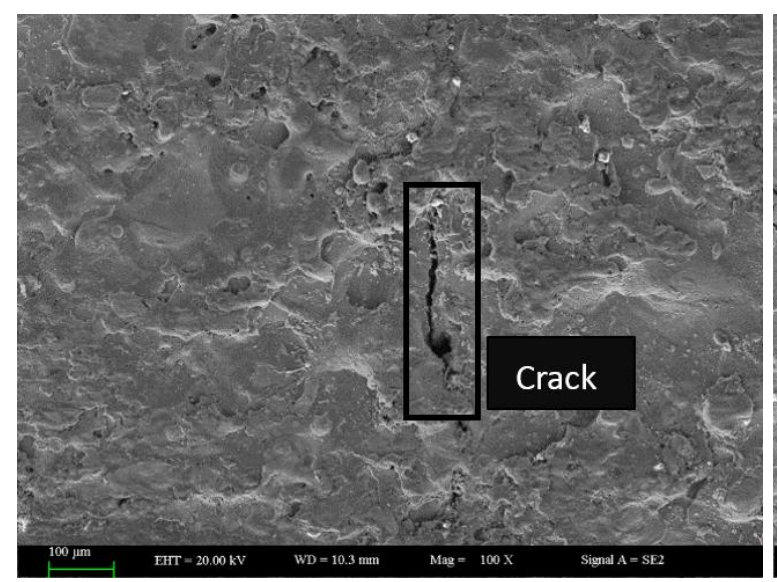

c) $\mathrm{D}=55 \%$

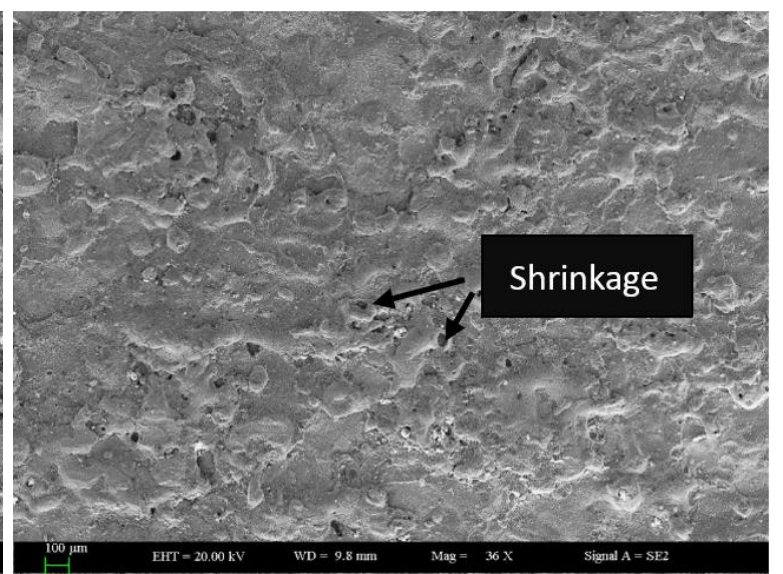

b) $\mathrm{D}=25 \%$

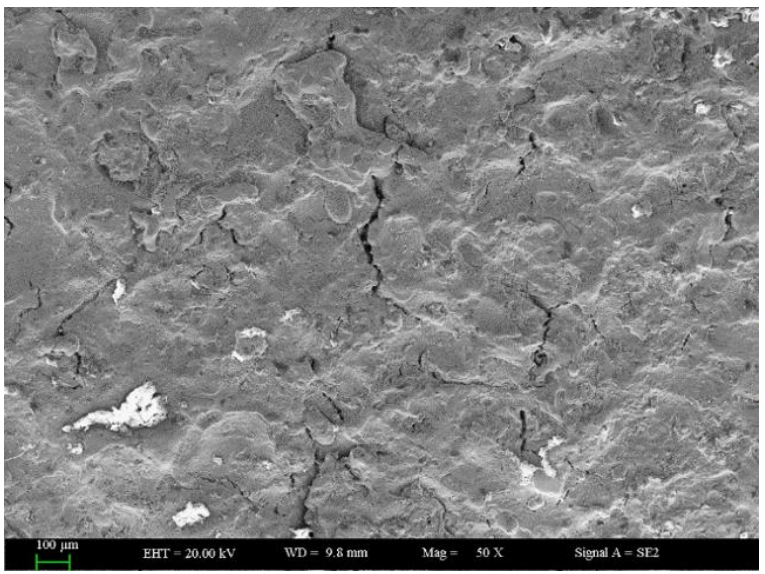

d) $\mathrm{D}=85 \%$

Figure 1. SEM of different duty cycles

The workpiece fits and starts, the inner material organization uneven expansion and contraction, surface of workpiece material is subjected to tension - compression stress and thermal effect. When the stress is greater than the ultimate strength of the material, that will cause local damage, and microcracks is a concentrated expression of local damage.

Analysis of Machining Section. Scanning the cross-section of specimen by electron microscope, in the top surface of the workpiece can be clearly found to melt solidified layer (Fig.2a). The reason is that in the pulsed arc process, surface melted by the instantaneous high temperature of arc discharge, re-solidified bonding to the surface by the working fluid rapid cooling. But melting solidified layer is usually rough, low toughness, connected with the base is not solid, prone to cracks, this character of engineering applications is often undesirable[11]. Generally, there are exist heat-affected layer, HAZ of metallic material is not melted, but the material is subjected to high temperature, the microstructure of the material changes, but for GH4169 superalloy specimens was not found HAZ, the reason is the lower thermal conductivity, making the temperature generated by pulsed arc discharge distribution on the surface only.

Melting solidified layer attaches to substrate unevenly (Fig.2b, Fig.2c Fig.2d), compared the distribution of recast layer at $100 \mathrm{HZ}, 400 \mathrm{HZ}, 700 \mathrm{HZ}$, discovered that thickness of recast layer increases with the increase of pulse frequency. With the increase of the pulse frequency, the workpiece region get more enery, resulting in more molten material so that the thickness of the recast layer is increase. 


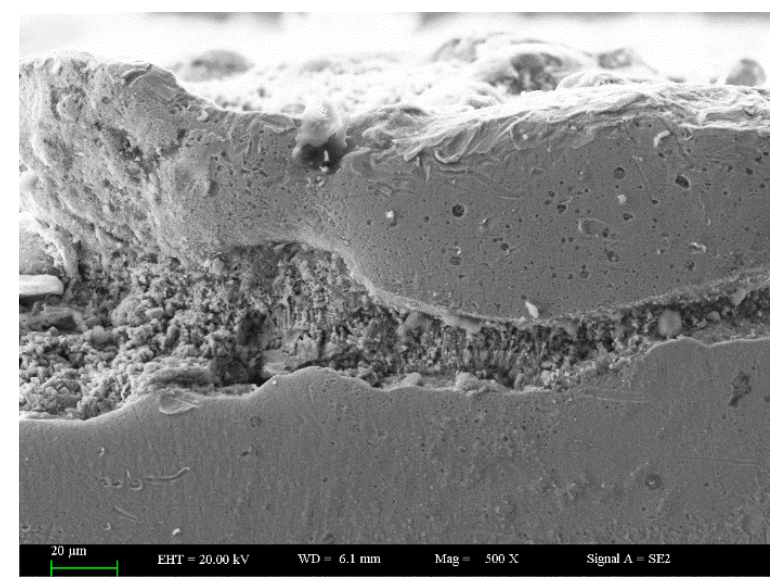

a) recast layer binding the substrate

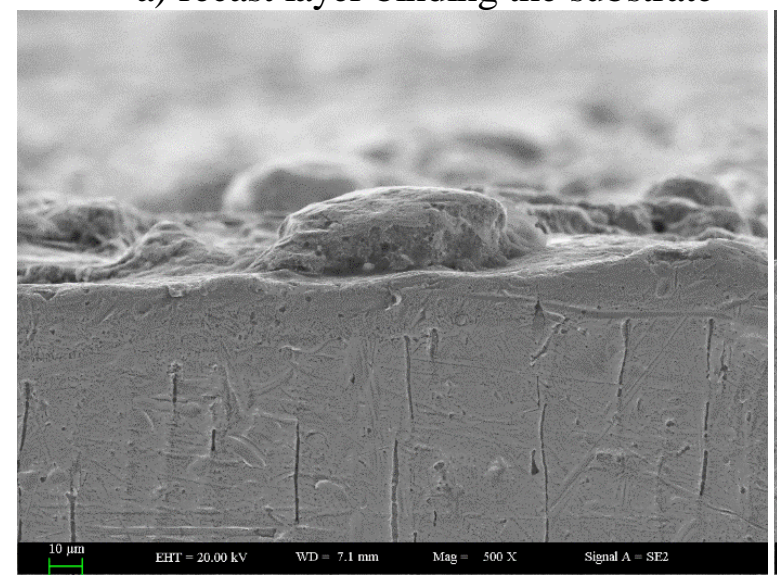

c) $\mathrm{f}=400 \mathrm{HZ}$

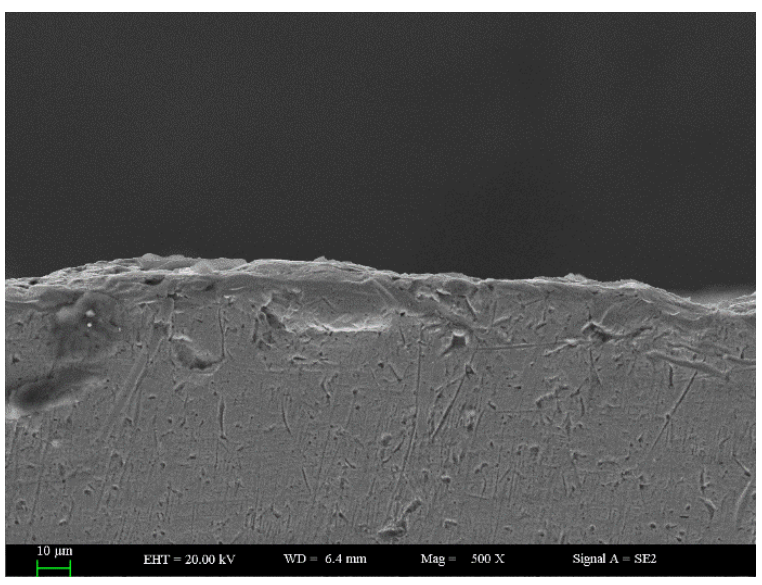

b) $\mathrm{f}=100 \mathrm{HZ}$

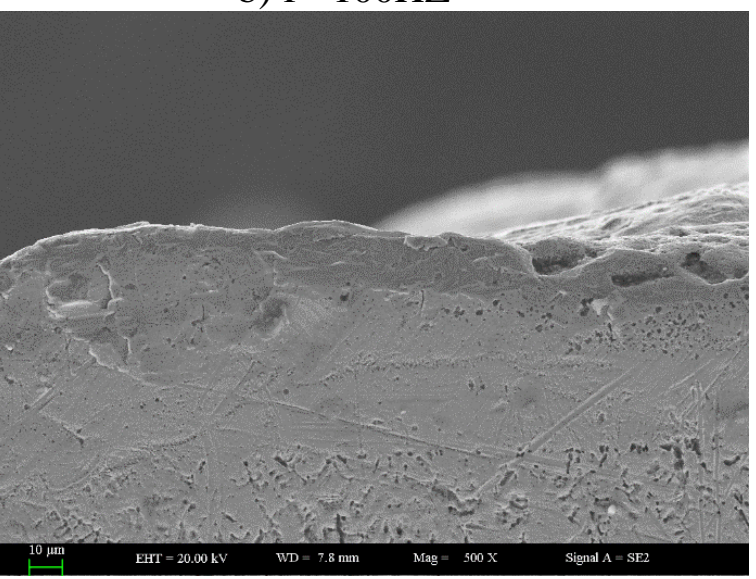

d) $\mathrm{f}=700 \mathrm{HZ}$

Figure 2. SEM of cross-section at different frequency processing

Surface Roughness. Fig.3a shows maintain the duty cycle at 50\%, surface roughness change with pulse frequency. Fig. $3 \mathrm{~b}$ is maintain the frequency at $400 \mathrm{HZ}$, the roughness change with the duty cycle. Figure a, roughness changes smoothly between $400 \sim 700 \mathrm{HZ}$, roughness lower, no more than $20 \mu \mathrm{m}$; Figure b, polyline of $15-45 \%$ and $65-95 \%$ both little fluctuation, obtain a minimum roughness when the duty cycle is $55 \%$.

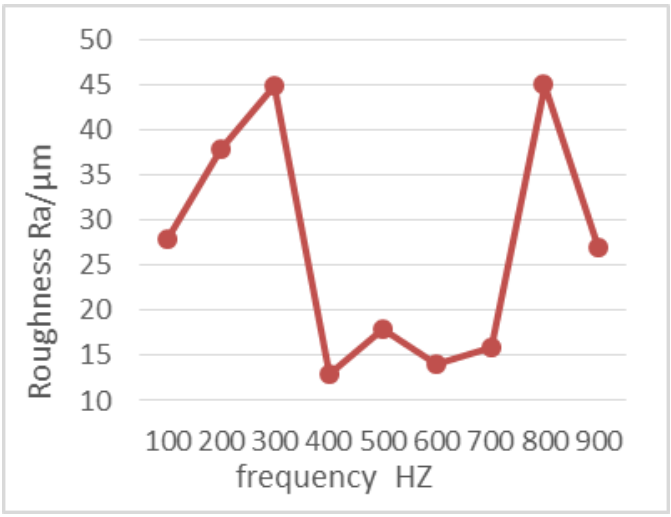

a) $\mathrm{D}=50 \%$

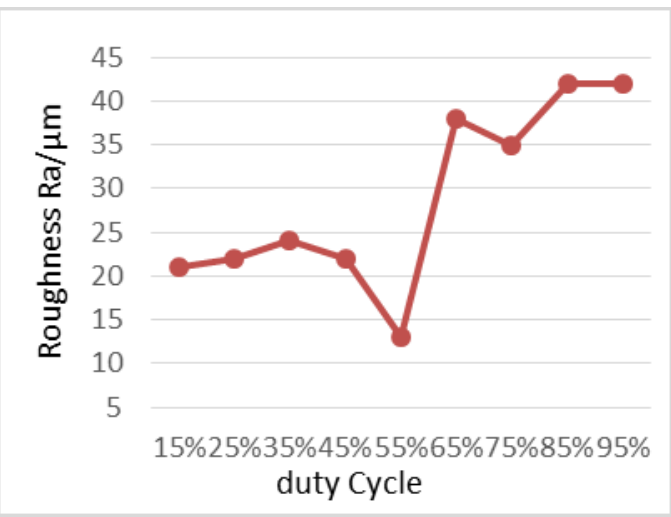

b) $\mathrm{f}=400 \mathrm{HZ}$

Figure 3. SEM of cross-section at different frequency processing

Based on the above, at a lower pulse frequency and duty cycle, material obtain less energy, and melting inadequate, working fluid difficult peeled off material from the base, the higher pulse frequency and duty cycle will melting a large number of material, part of the working fluid cooling the melted material to re-solidification, generate a large amount of recast layer, increased the surface roughness.

Microhardness. Fig.4 is a distance from the workpiece surface in the range of $0-350 \mu \mathrm{m}$, microhardness curve of specimen at different pulse frequency and duty ratio. 
Fig.4a shows the hardness change in the range from surface $0-50 \mu \mathrm{m}$, this region distribution of melting and solidification layer, when processing frequency is $100 \mathrm{HZ}$ and $400 \mathrm{HZ}$, their have similar hardness tendency, however, when working frequency increased to $700 \mathrm{HZ}$, its hardness changed greatly, the reason is with frequency increase the surface material get more energy, making the increase of molten material lead to the thicker recast layer. Fig. $4 \mathrm{~b}$ is microhardness curve of specimen at different duty ratio. tendency to change the hardness of the specimen when the duty cycle, with the disappearance of the recast layer, the hardness rapidly decreased to 450 ; when the duty ratio is $25 \%$ the change of hardness trend to relatively flat; when the duty cycle lower than $30 \%$, discharge get less effective, surface receives not enough energy, melted not completely. The molten material adhered to the substrate surface again, generate a thicker recast layer, meanwhile reduces the material removal rate significantly.

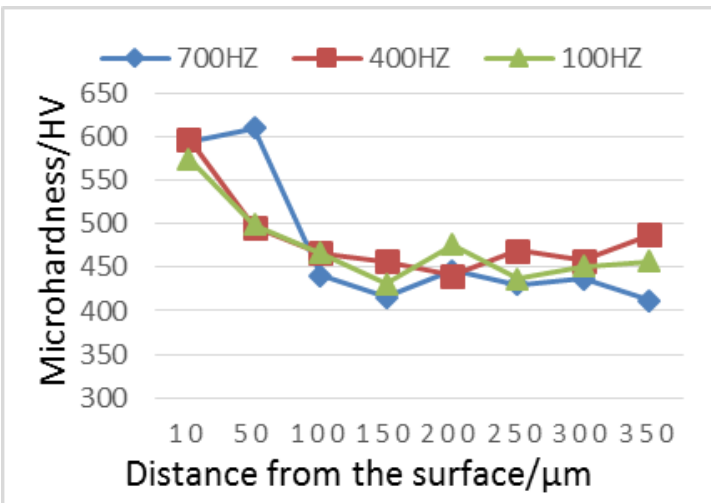

a) change the frequency

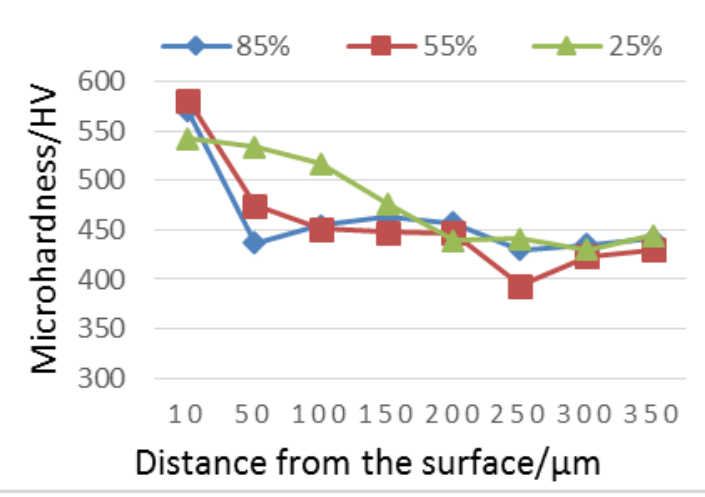

b) change the duty cycle

Figure 4. Microhardness of machined surface

\section{Conclusions}

Machined surface of pulsed arc machining nickel-based alloy composed of the pits, bumps, spherical droplet and tiny holes.

High duty cycle and pulse frequency makes surface have a lot of cracks, meanwhile the crack in the recast layer will extending into matrix.

Selecting the appropriate the combination of pulse frequency and duty ratio, can get better surface roughness.

Study shows that pulsed arc machining would produce recast layer, and it can affect the surface hardness, the thickness of the recast layer change with the frequency and duty cycle, high frequency and duty cycle prone to cracks, on the country, the low increased the thickness of recast layer.

\section{References}

[1] H. Xu, L. Gu and J. Chen: The International Journal of Advanced Manufacturing Technology, Vol. 79 (2015) No.5, p.937.

[2] L. Wang, Y. Wang and Y. Liu: International Journal of Minerals, Metallurgy and Materials, Vol. 20 (2013) No.9, p.861.

[3] Z.W. Du, Y. Chen and K .Zhou: The International Journal of Advanced Manufacturing Technology, Vol. 81 (2015) No.5, p.897.

[4] D.S. Wei, L. Shi and Y.R. Wang: Materials Science \& Engineering A, Vol. 569 (2013), p124.

[5] X.W. Kong, B. Li and Z.B. Jin: J. Mater. Sci. Technol, Vol. 27 (2011) No.12, p.1178.

[6] F.S. Qu, Z. Lu and F. Xing: Materials and Design, Vol. 39 (2012). p.151.

[7] C.H. Lang, J.P. Zhou and Z.J. Zhu: Modern Manufacturing Engineering, Vol. 12(2007), p. 92. 
[8] W. Zhao, L. Gu and H. Xu: Procedia CIRP, Vol.6 (2013), p.621.

[9] F.Z. Han, Y.X. Wang and M. Zhou: Int J Mach Tool Manu, Vol. 49(2009), p20.

[10] D.Thakur, B.Ramamoorthy and L.Vijayaraghavan: Indian J Eng mater Sci, Vol. 16(2009), p.44.

[11] S. Dhanabalan, K. Sivakumar and C. Sathiya Narayanan: Indian J Eng mater Sci, Vol. 20(2013), p.391. 